\title{
Evaluation of drug release kinetics from polymeric nanoparticles loaded with poorly water-soluble APIs
}

\author{
Ramona-Daniela PAVALOIU ${ }^{*}{ }^{1}$ Fawzia SHA'AT $1,{ }^{1,2}$ Cristina HLEVCA, ${ }^{1}$ Mousa SHA'AT, ${ }^{3}$ \\ Gabriela SAVOIU, ${ }^{1}$ and Sibel OSMAN ${ }^{4}$
}

\author{
${ }^{1}$ National Institute for Chemical-Pharmaceutical Research and Development - ICCF Bucharest, Vitan Avenue 112, $3^{\text {rd }}$ \\ District, Bucharest, Romania \\ ${ }^{2}$ Faculty of Applied Chemistry and Materials Science, University Politehnica of Bucharest, 1-7 Gheorghe POLIZU St., \\ 011061, Bucharest, Romania \\ ${ }^{3}$ Faculty of Pharmacy, Grigore T. Popa University of Medicine and Pharmacy, 11, Universitatii Street, Iasi, Romania \\ ${ }^{4}$ Department of Chemistry and Chemical Engineering, "Ovidius" University of Constanta, 124, Bd. Mamaia, 900521, \\ Constanta, Romania
}

\begin{abstract}
The aim of this research was to investigate the release behavior of a combination of two poorly water-soluble active pharmaceutical ingredients (APIs) from poly (D,L-lactide-co-glycolide) (PLGA) nanoparticles. Amlodipine besylate - AML, a calcium channel blocker, and valsartan - VAL, an angiotensin II receptor antagonist drug, were used as poorly water-soluble model drugs. PLGA nanoparticles loaded with AML-VAL (1:16 w/w) were obtained by nanoprecipitation using an amphiphilic block copolymer - Pluronic F127 as stabilizer. The drugs release from the PLGA nanoparticles was determined by a dialysis membrane method under sink conditions. Nanoparticles provided a slow release for both APIs and an attenuated burst effect compared to free drug. Five kinetics models such as Zero-order, Firstorder, Korsmeyer-Peppas, Higuchi and Hixson-Crowell were applied to predict drug release profiles. The Higuchi and Korsmeyer-Peppas models $\left(\mathrm{R}^{2}>0.97\right)$ best described physicochemical release phenomenon for each PLGA formulations.
\end{abstract}

Keywords: nanoparticles; amlodipine; valsartan; drug release.

\section{Introduction}

Low water solubility is often the main obstacle to the use of new drugs. This issue leads to a low bioavailability and therefore the drug's active components are not concentrated at the site of action and the treatment fails in vivo $[1,2]$.

Over the past decade, nanotechnology provides solutions to overcome the drawbacks of poorly watersoluble active pharmaceutical ingredients (APIs), including improved solubility, protection of APIs from external medium, controlled drug release, and targeted delivery that can result in an increase of therapeutic efficacy [3-8].

Biocompatible and biodegradable polymeric nanoparticles have been used for drug delivery applications. These are composed of either natural or synthetic materials $[9,10]$. One of the most successful polymers in the development of bio-based polymers is the poly-(lactic-co-glycolic acid) (PLGA) due to its numerous advantages, such as biodegradability, biocompatibility, and drug delivery system approval [11-13].

The study of release kinetics from nano-sized systems offers important data for the assessment of safety and therapeutic efficacy. Also, in vitro release kinetics can be correlated to the in vivo behavior of APIs through predictive mathematical models, resulting in a faster regulatory approval [14-16]. Therefore, the selection of a proper method to assess in vitro release kinetics of APIs from nanosystems is critical. Currently, there are no formal regulations or standards for the evaluation of drug release profiles of nanoparticles. However, a review of the literature shows that the drug release profiles can be obtained by various methods, such as flow cytometry, dialysis membrane, and sample and separate methods $[17,18]$.

In previous studies we evaluated several nanoformulations with different quantities of PLGA and we concluded that in the range of concentrations 5-60 mg PLGA and at a stirring rate of 1200-1500 rpm cardiovascular drugs loaded nanoparticles with good features can be obtained [19, 20]. However, the release behavior has only been studied for the nanoformulations with 5, 7.5 and $10 \mathrm{mg}$ PLGA [20]. The aim of this research was to investigate the release behavior of a combination of two poorly water-soluble APIs from PLGA nanoparticles with higher PLGA concentrations, in the range of 25-50 mg. Amlodipine besylate (AML), a calcium channel blocker, and valsartan (VAL), an angiotensin II receptor antagonist drug, were used as poorly water-soluble model drugs. PLGA nanoparticles loaded with AML-VAL were prepared and characterized in terms of entrapment efficiency (EE), size and polydispersity index (PDI). To evaluate the release behavior of AML-VAL the dialysis membrane approach was applied due to its simplicity and

\footnotetext{
* Corresponding author. E-mail address: pavaloiu_daniella@yahoo.com (Ramona-Daniela Pavaloiu)
} 
avoidance of separating released drug from the nanoparticles. Five kinetics models such as Zero-order, First-order, Korsmeyer-Peppas, Higuchi and HixsonCrowell were used to predict drug release mechanisms.

\section{Experimental}

\subsection{Materials}

Poly (lactic-co-glycolic acid) (50:50, MW $=30,000-$ $60,000 \mathrm{Da})$, amlodipine besylate, valsartan, and Pluronic F127: poly(ethylene glycol)-blockpoly(propylene glycol)-block-poly(ethylene glycol) were purchased from Sigma-Aldrich (Merck Group, Darmstadt, Germany). The in vitro drug release studies were performed in $0.1 \mathrm{M}$ phosphate buffer $\mathrm{pH}$ 7.4. All other chemicals were of analytical grade and used without further purification.

\subsection{Preparation of PLGA nanoparticles loaded with amlodipine-valsartan}

PLGA nanoparticles loaded with AML-VAL were prepared by nanoprecipitation method as described elsewhere [19, 20] using PLGA, as biodegradable polymeric and an amphiphilic block copolymer Pluronic F127 as stabilizer. The composition of prepared PLGA nanoparticles loaded with AML-VAL is presented in Table 1.

Table 1. Formulation of PLGA nanoparticles

\begin{tabular}{|c|c|c|c|c|}
\hline $\begin{array}{c}\text { Sample } \\
\text { Code }\end{array}$ & $\begin{array}{c}\text { maML:vAL } \\
(\mathbf{g} / \mathbf{g})\end{array}$ & $\begin{array}{c}\text { mPLGA } \\
(\mathbf{m g})\end{array}$ & $\begin{array}{c}\text { mPluronic } \\
(\mathbf{m g})\end{array}$ & $\begin{array}{c}\text { Stirring } \\
\text { rate } \\
(\mathbf{r p m})\end{array}$ \\
\cline { 1 - 4 } NP1 & $1: 16$ & 20 & 10 & \multirow{2}{*}{1200} \\
\cline { 1 - 3 } NP2 & $1: 16$ & 35 & 10 & \multirow{2}{*}{1200} \\
\cline { 1 - 3 } NP3 & $1: 16$ & 50 & 10 & \\
\hline
\end{tabular}

2.3. Characterization of PLGA nanoparticles loaded with amlodipine-valsartan

The prepared PLGA NPs were characterized in terms of entrapment efficiency (EE), particle size and polydispersity index (PDI). EE was evaluated using an indirect method as the ratio of the quantity of drugs present in nanoparticles and the initial quantity of drugs using a UV-Vis spectrophotometer (JASCO V-630 Spectrophotometer, Jasco International Co., Ltd., Tokyo, Japan). The quantity of drugs present in nanoparticles was assessed as the difference between the initial quantity of valsartan, respectively amlodipine, used for nanoparticle preparation and the quantity of valsartan (amlodipine) present in the supernatant. Particle size and polydispersity index were evaluated by Dynamic Light Scattering (DLS) using a particle size analyzer (Beckman Coulter N4 PCS Submicron, Coulter Company, France). Measurements were performed on diluted samples $(1: 20)$ at a scattering angle of $90^{\circ}$ and temperature of $25^{\circ} \mathrm{C}$. For each sample the mean values with standard deviations of 10 determinations were established. The values reported are the mean values with standard deviations for three replicate samples.

\subsection{In vitro drug release from PLGA nanoparticles}

The in vitro drug release studies from the PLGA nanoparticles were carried out using dialysis membrane method under sink conditions. A sample of PLGA nanoparticles loaded with AML-VAL $(1 \mathrm{~mL})$ was put in a dialysis cellulose bag with molecular weight cut-off: 14,000 Da (Sigma-Aldrich, Merck Group, Darmstadt, Germany). The ends of the dialysis bag were sealed and then it was immersed into $200 \mathrm{~mL} 0.1 \mathrm{M}$ phosphate buffer solution of $\mathrm{pH} 7.4$ as release medium. The whole system was kept under magnetic stirring $(150 \mathrm{rpm} / \mathrm{min})$ at $37^{\circ} \mathrm{C}$. Samples were withdrawn at predetermined intervals and the release medium was refilled with the same volume of fresh medium. The amounts of AML and VAL released were determined by measuring the absorbance at $365 \mathrm{~nm}$ (for AML) and $250 \mathrm{~nm}$ (for VAL) using an UV-VIS spectrophotometer (JASCO V-630 Spectrophotometer, Jasco International Co., Japan), according to the standard AML and VAL calibration curves. Also, a combination of free AML-VAL was subjected to the same release conditions as control. Release studies were performed in triplicate and average values with standard deviations were reported.

\section{Results and discussions}

\subsection{Characterization of PLGA nanoparticles - EE, size and PDI}

The EE, size and PDI of PLGA nanoparticles loaded with AML-VAL were displayed in Figure 1. All formulations presented good EE for both APIs, ranged from $65.35 \% \pm 0.11$ to $67.58 \% \pm 0.11$ for AML, and from $79.89 \% \pm 0.13$ to $80.10 \% \pm 0.14$ for VAL. Also, the formulations NP1, NP2, NP3 had nanometric size bellow $210 \mathrm{~nm}$ and a good size-homogeneity with a value of PDI below 0.11. These features are in agreement with results obtained in our previous studies [19, 20]. Also, Verma et al. [21] described loading of losartan, an angiotensin II receptor antagonist drug, in PLGA nanoparticles with a size below $300 \mathrm{~nm}$ and $87 \%$ entrapment efficiency. Jana et al. encapsulated felodipine, a calcium channel blocker, in PLGA monodispersed nanoparticles with a size of 0.216 to 0.442 and approximately $80 \%$ entrapment efficiency [22].

\subsection{In vitro release of $A M L$ and $V A L$ from PLGA nanoparticles}

The in vitro release of AML and VAL from PLGA nanoparticles was carried out in $0.1 \mathrm{M}$ phosphate buffer $\mathrm{pH} 7.4$ at $37{ }^{\circ} \mathrm{C}$, and the experimental data were presented in Figure 2 and Figure 3. The dissolution of free AML and VAL in $0.1 \mathrm{M}$ phosphate buffer $\mathrm{pH} 7.4$ at $37{ }^{\circ} \mathrm{C}$ reached a maximum after $6 \mathrm{~h}$ for $\mathrm{AML}$ and respectively $7 \mathrm{~h}$ for VAL. PLGA nanoparticles provided a slow release for both drugs compared to the dissolution of free drug in the same conditions. The formulation with the smaller amount of PLGA (20 mg) - NP1 had higher CDR, with release reaching a maximum of $72.7 \%$ for AML and $85.8 \%$ for VAL after 48 hours. As the proportion of polymer increased, the cumulative release of drugs decreased, for example the release from the formulation with medium amount of PLGA (35 mg) NP2 reached a maximum of $65.3 \%$ for AML and $78.1 \%$ for VAL in 48 hours, and the release from the formulation with higher amount of PLGA (50 mg) - NP3 reached a maximum of $57.3 \%$ for AML and $73.5 \%$ for VAL in the same time frame. Also, it can be observed 
that the release of drugs from the free AML-VAL exhibited a burst effect $(67.00 \pm 0.28 \%$ for AML, and $60.30 \pm 0.15 \%$ for VAL, respectively, released in the first 30 minutes), while in the case of active substances loaded in polymeric nanoparticles the burst effect was reduced $(25 \%$ for $\mathrm{AML}$ and $40 \%$ for VAL were released in the first 30 minutes from all PLGA-NPs).
The burst release of AML-VAL form PLGA nanoparticles could be explained by the diffusion of AML and VAL crystals adhered to the surface of the nanoparticles. The biphasic pattern of cardiovascular drugs release from PLGA NPs was in agreement with the results of other studies [22, 23].
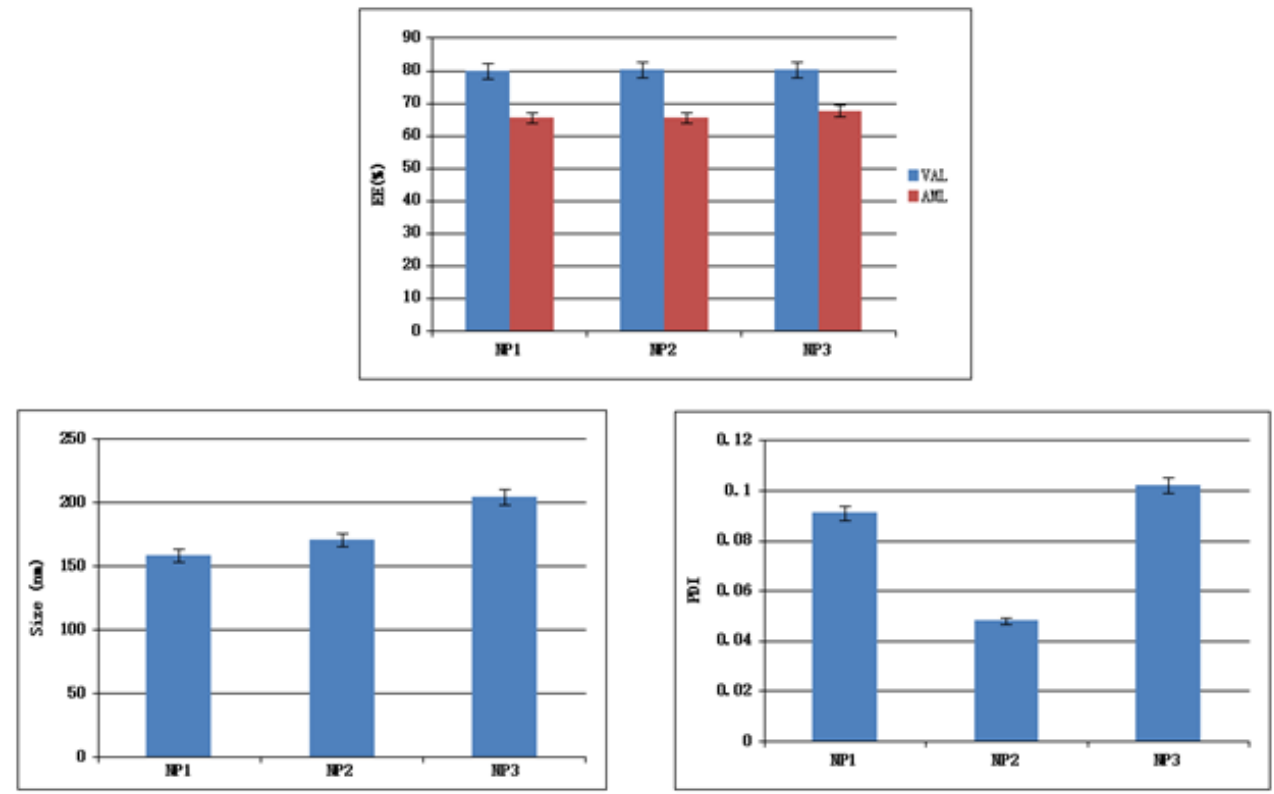

Figure 1. EE, size and PDI of PLGA nanoparticles loaded with AML-VAL

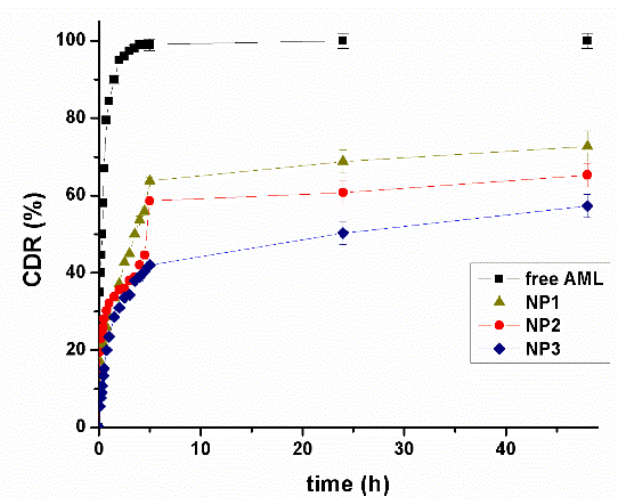

Figure 2. In vitro release profile of AML from PLGA nanoparticles

\subsection{Analysis of drug release mechanism}

In order to understand the release mechanism, experimental data obtained was fitted using KorsmeyerPeppas, Higuchi, Zero-order, First-order, and HixsonCrowell models:

$$
\begin{aligned}
& \frac{M(t)}{M(\infty)}=k_{K P} t^{n} \\
& \frac{M(t)}{M(\infty)}=k_{H} t^{1 / 2} \\
& \frac{M(t)}{M(\infty)}=k_{0} t
\end{aligned}
$$

$$
\begin{aligned}
& \frac{M(t)}{M(\infty)}=e^{-k_{1} t} \\
& \frac{M(t)}{M(\infty)}=\left[1-\left(1-k_{H C} t\right)^{3}\right]
\end{aligned}
$$

where $M(t)$ represents the amount of AML, respectively VAL released at time $t$ and $M(\infty)$ represents the total amount of AML, respectively $\mathrm{VAL}$ loaded in the polymeric nanoparticles; $\mathrm{k}_{0}, \mathrm{k}_{1}, \mathrm{k}_{\mathrm{H}}, \mathrm{k}_{\mathrm{KP}}$ and $\mathrm{k}_{\mathrm{HC}}$ are the constants of the Zero-order, the First-order, the Higuchi, the Korsmeyer-Peppas and the Hixson - Crowell models.

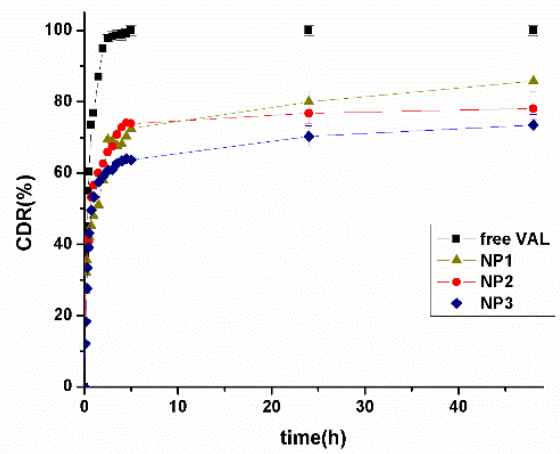

Figure 3. In vitro release profile of VAL from PLGA nanoparticles

Table 2. Correlation coefficient for various mathematical models - free versus entrapped AML release from PLGA nanoparticles

\begin{tabular}{|c|c|c|c|c|c|}
\hline \multirow{2}{*}{ Sample } & \multicolumn{5}{|c|}{ Correlation coefficient $\left(\mathbf{R}^{2}\right)$} \\
\cline { 2 - 6 } & Zero-order & First-order & Higuchi & Korsmeyer-Peppas & Hixson-Crowell \\
\hline Free AML & 0.8514 & 0.9680 & $-*$ & $-*$ & 0.9345 \\
\hline
\end{tabular}




\begin{tabular}{|c|c|c|c|c|c|}
\hline \multirow{2}{*}{ Sample } & \multicolumn{5}{|c|}{ Correlation coefficient $\left(\mathbf{R}^{\mathbf{2}}\right)$} \\
\cline { 2 - 6 } & Zero-order & First-order & Higuchi & Korsmeyer-Peppas & Hixson-Crowell \\
\hline NP1 & 0.9348 & 0.9509 & 0.9856 & 0.9836 & 0.9465 \\
\hline NP2 & 0.9106 & 0.9633 & 0.9759 & 0.9776 & 0.9410 \\
\hline NP3 & 0.8964 & 0.9282 & 0.9842 & 0.9833 & 0.9200 \\
\hline
\end{tabular}

* Condition for application of Korsmeyer-Peppas and Higuchi model was not met $(\mathrm{M}(\mathrm{t}) / \mathrm{M}(\infty)<2 / 3)$

Table 3. Correlation coefficient for various mathematical models - free versus entrapped VAL release from PLGA nanoparticles

\begin{tabular}{|c|c|c|c|c|c|}
\hline \multirow{2}{*}{ Sample } & \multicolumn{5}{|c|}{ Correlation coefficient $\mathbf{( R}^{\mathbf{2}}$ ) } \\
\cline { 2 - 6 } & Zero-order & First-order & Higuchi & Korsmeyer-Peppas & Hixson-Crowell \\
\hline Free VAL & 0.7207 & 0.9762 & $-*$ & $-*$ & 0.9548 \\
\hline NP1 & 0.8780 & 0.8654 & 0.9555 & 0.9879 & 0.8320 \\
\hline NP2 & 0.6534 & 0.7413 & 0.9645 & 0.9886 & 0.7929 \\
\hline NP3 & 0.8890 & 0.8264 & 0.9677 & 0.9618 & 0.7129 \\
\hline
\end{tabular}

* Condition for application of Korsmeyer-Peppas and Higuchi model was not met (M (t) / M ( $\infty)<2 / 3)$

In Table 2 and Table 3 are listed the correlation coefficients and parameters of mathematical models used for fitting the experimental data of AML and VAL release from loaded polymeric nanoparticles in comparison with drugs solubilization in PBS. The correlation coefficient $\left(\mathrm{R}^{2}\right)$ was chosen to compare the models, where a value closer to 1 means a better correlation.

The solubility curve of both amlodipine and valsartan can best be described by an exponential equation, with $\mathrm{R}^{2}=0.9680$ for $\mathrm{AML}$ and $\mathrm{R}^{2}=0.9762$ for VAL. For PLGA-based polymeric nanosystems, it was observed that the release of AML and VAL from all samples is best described by the Higuchi model and the Korsmeyer-Peppas model $\left(\mathrm{R}^{2}>0.95\right)$. The release behavior of nanoparticles with low PLGA concentrations obtained in our previous research [20] was also best described by Higuchi model, and the Korsmeyer-Peppas model was not applied. In the Korsmeyer-Peppas model, $\mathrm{k}_{\mathrm{KP}}$ is a constant that depends on the characteristics of the system, and the coefficient $n$ shows the nature of the release mechanism. When $n \leq$ 0.5 , the release is dominated by the Fickian diffusion mechanism; if $n$ is between 0.5 and 1 then the release follows the mechanism of an abnormal diffusion (nonFickian diffusion), and if $n>1$, the release is based on a complex transport mechanism (super-case-II transport). In the Higuchi's model, $\mathrm{k}_{\mathrm{H}}$ is a constant proportional to the burst release rate of the release process. The parameters of the Korsmayer-Peppas and Higuchi models for the analysis of AML and VAL release behavior from PLGA polymeric nanoparticles are presented in Table 3 and Table 4. For all the samples the values of the $n$ coefficient are below 0.5 , indicating a Fickian diffusion.

Comparing with our previous study [20] the samples with high PLGA content $(20-50 \mathrm{mg})$, the coefficient $n$ is less than 0.5, indicating Fickian diffusion, while the other samples (5-10 mg PLGA) in this range between 0.5 and 1 , indicating a non-Fickian diffusion. Nanoformulations with high PLGA content showed a lower value for $\mathrm{k}_{\mathrm{H}}$ than the other polymeric nanoformulations, indicating a less intense burst effect. Also, Jana et al. found the $n$ value, the parameter of Korsmeyer-Peppas model, less than 0.5 indicating that the release mechanism of felodipine from the PLGA nanoparticles was diffusion controlled [22].

Table 4. Parameters of Korsmayer-Peppas and Higuchi models for the release behavior analysis of AML from PLGA nanoparticles

\begin{tabular}{|c|c|c|c|}
\hline \multirow{2}{*}{ Sample } & \multicolumn{2}{|c|}{ Korsmayer-Peppas } & Higuchi \\
\cline { 2 - 4 } & $n$ & $k_{K P}$ & $k_{H}$ \\
\hline NP1 & 0.1862 & 27.8001 & 14.5410 \\
\hline NP2 & 0.1943 & 31.6602 & 17.2252 \\
\hline NP3 & 0.1957 & 22.5900 & 26.1869 \\
\hline
\end{tabular}

Table 5. Parameters of Korsmayer-Peppas and Higuchi models for the release behavior analysis of VAL from PLGA nanoparticles

\begin{tabular}{|c|c|c|c|}
\hline \multirow{2}{*}{ Sample } & \multicolumn{2}{|c|}{ Korsmayer-Peppas } & Higuchi \\
\cline { 2 - 4 } & $n$ & $k_{K P}$ & $k_{H}$ \\
\hline NP1 & 0.2311 & 49.0250 & 28.8182 \\
\hline NP2 & 0.2765 & 55.4978 & 39.2557 \\
\hline NP3 & 0.2644 & 55.8015 & 39.1066 \\
\hline
\end{tabular}

\section{Conclusions}

Nanoparticles provided a slow release for both APIs and an attenuated burst effect compared to free drug. Five kinetics models such as Zero-order, First-order, Korsmeyer-Peppas, Higuchi and Hixson-Crowell were applied to predict drug release profiles. The Higuchi and Korsmeyer-Peppas models $\left(\mathrm{R}^{2}>0.95\right)$ best described physicochemical release phenomenon for each PLGA formulations. All the nanoparticles had the values of $n$ coefficient below 0.5 , indicating a Fickian diffusion.

Acknowledgement: This work was financially supported by ANCSI through PN 19410401.

\section{Conflict of interest}

Authors declare no conflict of interest. 


\section{References}

[1]. J. Cardot, E. Beyssac, M. Alric, In vitro-in vivo correlation: Importance of dissolution in IVIVC. Dissolution Technol. 14 (2007) 15. Doi: dx.doi.org/10.14227/DT140107P15.

[2]. S. D'Souza, A review of in vitro drug release test methods for nano-sized dosage forms, Adv. Pharm. 2014 (2014) 1-12. https://doi.org/10.1155/2014/304757.

[3]. J. Weng, H. Tong, S. F. Chow, In Vitro Release Study of the Polymeric Drug Nanoparticles: Development and Validation of a Novel Method. Pharmaceutics 12 (2020) 732. https://doi.org/10.3390/pharmaceutics12080732.

[4]. H.D. Williams, N.L. Trevaskis, S.A. Charman, R.M. Shanker, W.N. Charman, C.W. Pouton, C.J.H. Porter, Strategies to address low drug solubility in discovery and development, Pharmacolical Reviews 65 (2013) 315-499. Doi: 10.1124/pr.112.005660.

[5]. K. Soppimath, T.M. Aminabhavi, A.R. Kulkarni, W.E. Rudzinski, Biodegradable polymeric nanoparticles as drug delivery devices, Journal of Controlled Release 70 (2001) 1-20. Doi: 10.1016/s0168-3659(00)00339-4.

[6]. S. Nimesh, R. Manchanda, R. Kumar, A. Saxena, P. Chaudhary, V. Yadav, S. Mozumdar, R. Chandra, Preparation, characterization and in vitro drug release studies of novel polymeric nanoparticles, International Journal of Pharmaceutics 323 (2006) 146- 152. Doi: 10.1016/j.ijpharm.2006.05.065.

[7]. J.U.A.H. Junghanns, R.H. Müller, Nanocrystal technology, drug delivery and clinical applications, International Journal of Nanomedicine 3 (2008) 295-309. Doi: 10.2147/ijn.s595.

[8]. P. Khadka, J. Ro, H. Kim, I. Kim, J.T. Kim, H. Kim, J.M. Cho, G. Yun, J. Lee, Pharmaceutical particle technologies: an approach to improve drug solubility, dissolution and bioavailability, Asian Journal of Pharmaceutical Sciences 9 (2014) 304316. https://doi.org/10.1016/j.ajps.2014.05.005.

[9]. F. Danhier, E. Ansorena, J. M. Silva, R. Coco, A. Le Breton, V. Préat, PLGA-based nanoparticles: An overview of biomedical applications, Journal of Controlled Release, 161 (2012) 505-522. Doi: https://doi.org/10.1016/j.jconrel.2012.01.043.

[10]. J. Jeevanandam, A. Barhoum, Y.S. Chan, A. Dufresne, M.K. Danquah. Review on nanoparticles and nanostructured materials: history, sources, toxicity and regulations, Beilstein Journal of Nanotechnology 9 (2018) 1050-1074. Doi: 10.3762/bjnano.9.98.

[11]. T. Matoba, J. Koga, K. Nakano, K. Egashira, H. Tsutsui, Nanoparticle-mediated drug delivery system for atherosclerotic cardiovascular disease, Journal of Cardiology 70 (2017) 206-211. https://doi.org/10.1016/j.jjcc.2017.03.005.

[12]. O. Pechanova, E. Dayar, M. Cebova, Therapeutic potential of polyphenols-loaded polymeric nanoparticles in cardiovascular system, Molecules 25 (2020) 3322.

Doi: $10.3390 /$ molecules25153322.

[13]. D. Essa, P.P.D. Kondiah, Y.E. Choonara, V. Pillay, The design of poly(lactide-co-glycolide) nanocarriers for medical applications, Frontiers in Bioengineering and Biotechnology 8 (2020) 48 . Doi: 10.3389/fbioe.2020.00048.

[14]. L. Nothnagel, M.G. Wacker, How to measure release from nanosized carriers? European Journal of Pharmaceutical Sciencies 120 (2018) 199-211. Doi: 10.1016/j.ejps.2018.05.004.

[15]. M. Yu, W. Yuan, D. Li, A. Schwendeman, S.P. Schwendeman, Predicting drug release kinetics from nanocarriers inside dialysis bags, Journal of Controlled Release 315 (2019) 23-30. Doi: 10.1016/j.jconrel.2019.09.016.

[16]. S. Modi, B.D. Anderson, Determination of drug release kinetics from nanoparticles: Overcoming pitfalls of the dynamic dialysis method, Mol. Pharm. 10 (2013) 3076-3089. https://doi.org/10.1021/mp400154a.

[17]. M. Yu, W. Yuan, D. Li, A. Schwendeman, S.P. Schwendeman, Predicting drug release kinetics from nanocarriers inside dialysis bags, Journal of Controlled Release 315 (2019) 23-30. https://doi.org/10.1016/j.jconrel.2019.09.016.

[18]. Y. Zambito, E. Pedreschi, G. Di Colo, Is dialysis a reliable method for studying drug release from nanoparticulate systems?-A case study, Int. J. Pharm. 434 (2012) 28-34. https://doi.org/10.1016/j.ijpharm.2012.05.020.

[19]. F. Sha'at, R.D. Pavaloiu, D.C. Salceanu, C. Hlevca, G. Nechifor, Formulation of Polymeric Multicomponent Systems Containing Cardiovascular APIs, Materiale Plastice 55 (2018) 121-123. Doi: 10.37358/MP.18.1.4976.

[20]. F. Sha'at, R.D. Pavaloiu, D.C. Salceanu, C. Hlevca, G. Nechifor, Evaluation of AML-VAL nanoparticles as combined therapy in cardiovascular disease, Materiale Plastice 54 (2018) 299-302. Doi: 10.37358/MP.18.3.5017.

[21]. A. Verma, S. Ratnawat, A. Gupta, S. Jain, PLGA nanoparticles for delivery of losartan potassium through intranasal route: development and characterization, Int. J. Drug Dev. \& Res. 5 (2013) 185- 196.

[22]. U. Jana, A.K. Mohanty, S.L. Pal, P.K. Manna, G.P. Mohanta, Felodipine loaded PLGA nanoparticles: preparation, physicochemical characterization and in vivo toxicity study, Nano Convergence 1 (2014) 31. https://doi.org/10.1186/s40580-014-0031-5.

[23]. M.L. Zweers, G.H. Engbers, D.W. Grijpma, J. Feijen, In vitro degradation of nanoparticles prepared from polymers based on DL-lactide, glycolide and poly(ethylene oxide), Journal of Controlled Release 100 (2004) 347-356. https://doi.org/10.1016/j.jconrel.2004.09.008.

Received: 03.06.2021

Received in revised form: 18.11.2021

Accepted: 19.11.2021 